\title{
Programas psicosociales y educativos en residencias y centros de día para personas mayores en Andalucía. Situación actual y posibles mejoras
}

\author{
Belén López Moya | Psicóloga Clínica en el Servicio Madrileño de Salud | Universidad de \\ Granada \\ Recepción: 22 de septiembre de 2015 | Revisión: 30 de septiembre de 2015 | Aceptación/Publicación: 7 de octubre de 2015 \\ Correspondencia: belenlmpsico@hotmail.com \\ Citar: López Moya, B. (2015). Programas psicosociales y educativos en residencias y centros de día para personas mayores en \\ Andalucía. Situación actual y posibles mejoras. ReiDoCrea, 4, 277-291. [http://hdl.handle.net/10481/38408]
}

\begin{abstract}
Resumen: El presente trabajo expone parte de una investigación llevada a cabo con el objetivo de conocer cómo son en la práctica los programas de actividades en residencias y centros de día para personas mayores en Andalucía, así como la participación, satisfacción y necesidades de las personas mayores con los mismos, para así proponer posibles mejoras que repercutan en una mayor calidad de vida de este colectivo. En el estudio han participado 62 personas mayores, usuarias de residencias y centros de día de Andalucía. Se trata de un estudio transversal descriptivo. Como instrumento se ha utilizado una entrevista semiestructurada.
\end{abstract}

Palabras claves: Programas de educación | Calidad de vida

Psychosocial and educational programs in nursing homes and day centers for the elderly in Andalusia. Current situation and possible improvements

\begin{abstract}
This paper exposes part of an investigation carried out in order to know how are in practice programmers in nursing homes and day center for elderly people in Andalusia, as well as participation, satisfaction and needs of older persons with the same, to propose possible improvements affecting a greater quality of life for this group. The study involved 62 elderly people, users of residences and day center in Andalusia. It is a cross-sectional descriptive study. As an instrument has been used a semi-structured interview.
\end{abstract}

Key words: Educational programs | Quality of life

\section{Introducción}

Se está produciendo un crecimiento cuantitativo de las personas mayores en el conjunto de la población y se observa al mismo tiempo un cambio en las actitudes y condiciones psicosociales de las personas mayores. Por otro lado han cambiado las formas tradicionales de familia así como el mundo del trabajo y los avances tecnológicos, por lo que se hace necesario replantear los roles, intereses, motivaciones y posibilidades de desarrollo personal de los mayores. El aumento de la esperanza de vida es un logro social, pero es necesario adaptar los recursos y políticas sociales a esta realidad que se está dando, donde la población de personas mayores está en aumento.

"Según datos del Libro Blanco sobre el Envejecimiento elaborado por el IMSERSO (2011), la población española mayor de 65 años, en Enero de 2010 era de 7,9 millones de personas, lo que representa un $16,9 \%$ del total de la población. En los últimos 45 años, el porcentaje de personas mayores en nuestro país se ha duplicado, pasando del $7 \%$ al $14 \%$, una tasa que, Francia tardó 115 años en alcanzar y Suecia 85 años.

A modo de reseña, en el año 2003 los datos demográficos nacionales (INE) señalaban que la población total era de 42.717 .064 personas y había contabilizadas en España 7.276 .620 personas de 65 y más años, lo que representa el $17 \%$ de toda la población. Las previsiones de Naciones Unidas estiman que, en el año 2050, España será el país 
más viejo del mundo con más de 16 millones de personas mayores, es decir, más de un $30 \%$ del total.

La población anciana se ha hecho visible en el mundo actual, de tal modo que sus necesidades y problemas comienzan a ser, sin excepción, las necesidades y problemas de todos. El acceso a la cultura y a la educación es vital para que las personas mayores reencuentren su identidad, puedan llevar a la realidad sus capacidades y tengan cubiertas sus necesidades. La educación reencuentra al adulto mayor con la ilusión y el gusto por el conocimiento. Estas ganas por aprender proporcionan confianza en uno mismo, libertad y transformación personal. Las actividades educativas en las personas mayores sirven de apertura social, permitiendo a la persona fortalecer o reformular su identidad personal y social.

El presente artículo pretende analizar las acciones socioeducativas presentes en los centros de día y residencias de mayores en Andalucía, para realizar propuestas de mejora de estos programas y actividades que redunden en una mejor calidad de vida de las personas mayores.

Los objetivos que nos proponemos son:

- Conocer las actividades llevadas a cabo en residencias y centros de día para personas mayores.

- Conocer el grado de participación de los mayores en las actividades que se les ofrecen desde estos servicios.

- Conocer la satisfacción de los mayores con estas actividades y los motivos por los que están o no satisfechos.

- Conocer qué beneficios encuentran los mayores en las actividades en que participan y qué les motiva a participar.

- Conocer otras actividades de interés y necesidades socioeducativas de las personas mayores en centros de día y residencias.

- Saber si la actividad de los participantes era diferente o no antes de acudir al centro de día o residencia.

- Conocer el estado de ánimo general de las personas mayores usuarias de centros de día y residencias.

- Plantear posibles actividades u objetivos para mejorar el estado emocional y psicológico de las personas mayores que acuden a centros de día y residencias.

Conocer esta información nos permitirá poder ofertar a este colectivo unos programas más adaptados a sus necesidades, teniendo en cuenta su heterogeneidad como grupo. Dentro de los factores importantes que favorecen la calidad de vida en los mayores, están los programas de actividades, terapéuticos, de ocio y educativos. Por este motivo nos parece importante este estudio, ya que conocer las variables que favorecen la mejora de los mismos supondrá una mejor adaptación a las necesidades de los mayores y una mejora de su calidad de vida. Fernández Ballesteros investigó en una muestra representativa de españoles, cuáles eran los "ingredientes" principales que constituían su calidad de vida y encontró los siguientes en este orden: estado de salud, habilidades funcionales, recursos económicos, relaciones familiares y sociales, actividades de la vida diaria y recreativas, servicios sociales y de salud, satisfacción vital, recursos culturales y entorno. (Fernández Ballesteros 1993, 1997) 
Envejecer bien, no sólo en lo relacionado con la salud, sino de manera integral es un concepto que comienza a adquirir gran importancia en la sociedad, por lo que el ámbito de la educación social debe comenzar a tenerlo presente y trabajar en esta línea. "La vejez, como otras etapas de la vida, es además de una cuestión biológica y psicológica, una construcción social" (Bazo, 2005:14). Y como construcción social es necesario que las personas mayores participen en la sociedad; sobre todo, si tenemos en cuenta su destacable peso poblacional. Participar de manera plena es contribuir en la elaboración y realización del proyecto global de sociedad: una sociedad para todas las edades. (Lema del Año Internacional de las Personas Mayores, 1999)

En cuanto al perfil de persona mayor en España se debe de tener presente el bajo nivel educativo que suele tener, lo que supone una peor adaptación al entorno. Esto unido al posible deterioro que puede ir asociado a la edad, lo convierte en un grupo con riesgo de marginación.

En cuanto a los programas psicoeducativos, nadie se atrevería a discutir sobre su necesidad. El concepto de educación es muy amplio y como bien sabemos la educación es algo intrínseco al ser humano, abarca todas las ramas del saber y todos los aspectos de la persona. Para la Pedagogía Social, la Educación en personas mayores resulta un ámbito de actuación destacado y en expansión, que por otra parte tiene una repercusión directa en el aumento de su calidad de vida (Bedmar y Montero, 2009).

Uno de los problemas con los que se enfrentan los profesionales que trabajan en las residencias de personas mayores son los efectos que tiene la institucionalización sobre los/as ancianos/as. En muchos casos el anciano se valora negativamente a sí mismo, disminuye la capacidad de adaptación y la frecuencia de los contactos sociales, así como de la actividad en general, y se modifica la noción de tiempo. Todo esto dificulta la participación del anciano en las programas y actividades. Todo esto sería extrapolable a los profesionales y personas mayores en centros de día, donde los usuarios pasan gran parte del día en el centro.

Las residencias y centros de día para personas mayores deben reunir una serie de condiciones físicas, ambientales y de personal, capaces de dar respuesta a los intereses y necesidades de los residentes. La dimensión educativa desempeña un papel relevante en la planificación de los programas, proyectos y actividades que se realicen en las mismas.

De las diferentes definiciones que hay del concepto de residencia nos parece especialmente completa y acertada la de Rodríguez, que la define como "centro gerontológico abierto, de desarrollo personal y atención socio-sanitaria multiprofesional en el que viven temporal y permanentemente personas mayores con algún grado de dependencia" (Rodríguez, 1999). Otra definición de residencia es la del Estatuto Básico de Centros del IMSERSO: "establecimientos destinados a servir de vivienda permanente y común a personas de la $3^{a}$ edad, en los que se presta una asistencia integral y continuada, a quienes no pueden satisfacer estas necesidades por otros medios" (IMSERSO, 1985). Como mencionan Kaufmann y Frías, una residencia debería tratar de crear un ambiente lo más parecido al familiar, y que la persona mayor identifique ésta como su hogar (Kaufmann y Frías, 1996).

Respecto a los centro de día, se puede definir como un recurso social intermedio, un centro gerontológico socioterapéutico y de apoyo a la familia que durante el día presta 
atención a las necesidades básicas, terapéuticas y sociales de la persona mayor dependiente, promoviendo su autonomía y una permanencia adecuada en su entorno habitual.

No existe información específica suficiente sobre la manera de abordar la educación en personas mayores en residencias y centros de día, por lo que este estudio trata de dar respuesta en primer lugar a cómo es la situación actual en las residencias y centros de día de mayores en este sentido y hacer propuestas para la mejora de esta situación. Las conclusiones que se saquen de este estudio contribuirán a perfeccionar el modo de intervención de profesionales dedicados a la programación y realización de actividades psicosociales y educativas con mayores en estos centros y a la mejora de los proyectos que se lleven a cabo, para que sean lo más acordes posible a los intereses y necesidades de las personas mayores.

\section{Metodología}

\section{Participantes}

En el estudio han participado 62 personas mayores, de las cuales 53 son usuarias de centros de día y 9 de residencias para personas mayores en Andalucía. En cuanto al sexo, 44 son mujeres y 18 hombres. La edad está entre los 66 y los 95 años. El intervalo entre 66 y 75 años supone un 33,9\%, frente al 50,8 \% de 75-85 años, el 9,7 $\%$ de $86-90$ años y un 3,2 \% con más de 90 años.

Respecto al estado civil el $14,5 \%$ de los participantes están casados, un $3,2 \%$ divorciados, un $22,6 \%$ solteros y un $61,3 \%$ viudos/as, siendo por tanto este último el estado civil el más prevalente de la muestra.

En cuanto al nivel de estudios de los participantes, el $41,9 \%$ no tienen estudios, el $46,8 \%$ sólo primarios, el $6,5 \%$ estudios medios y el $4,8 \%$ estudios superiores.

En relación a la ocupación que han tenido a lo largo de su vida, un 32,3\% de la muestra ha sido ama de casa, todas mujeres, dedicadas al cuidado de los hijos y a sus labores. El 8,1\% de los participantes se ha dedicado a la agricultura, el 6,4\% al comercio, el $37,1 \%$ a otras profesiones (ganadería, costura, sanidad, cura, cocina, carpintería, hostelería, administración, construcción, etc.) y un 8,1 \% no ha respondido.

Respecto al tiempo que llevan siendo usuarios del centro de día o residencia, un $3,2 \%$ lleva menos de 3 meses, un 1,6\% entre 3 y 6 meses, un $25,8 \%$ de 6 a 12 meses, un $14,5 \%$ de 18 a 24 meses y un $40,3 \%$ más de 24 meses.

\section{Instrumento}

Para la recogida de la información se ha utilizado una entrevista semiestructurada con preguntas abiertas y cerradas.

La entrevista consta de 37 preguntas mediante las que se trata de recoger información de diferente índole: variables sociodemográficas (sexo, edad, nivel educativo, estado civil, etc.), satisfacción con relaciones sociales (familia, otros residentes, personal del centro...), satisfacción con el centro y diferentes factores de éste (instalaciones, atención recibida...), estado de ánimo... así como diferentes preguntas específicas 
sobre el tema principal de nuestra investigación (las actividades realizadas en el centro, participación en ellas, preferencias e intereses personales, etc.)

Por último se ha dejó un espacio para poder reflejar otros datos de interés sobre la salud física, déficit sensoriales, ayudas técnicas u otros aspectos que la persona mayor entrevistada considerara relevante.

\section{Procedimiento}

El primer paso fue ponernos en contacto con los directores de los centros que han participado en el estudio y plantearles la posibilidad de realizar una serie de entrevistas a las personas mayores del centro, describiéndoles el objetivo de nuestro estudio. Tras su aceptación se fijaron los días en que se realizarían.

Las entrevistas personales para la recogida de información se llevaron a cabo en los diferentes centros, con la ayuda de colaboradores que participaron en esta fase del estudio.

Antes de realizar las entrevistas se les pasó el test de Pfeiffer (SPSMQ) para descartar deterioro cognitivo importante, ya que las personas con deterioro no podrían responder de manera adecuada a las preguntas que les planteaban.

Antes de comenzar con la entrevista, se le pedía a la persona mayor su colaboración, explicándole el motivo de la misma y asegurándole el anonimato. Todas las personas participaron de manera voluntaria. Una vez se les explicaba el modo en que se llevaría a cabo la entrevista, se comenzaban a plantear los diferentes ítems. Se trató en todo momento que la persona diera la respuesta de manera espontánea, sin ofrecerle las diferentes opciones de respuesta (en las preguntas con respuestas estructuradas), para así obtener una información más rica y de mayor utilidad.

En muchas de las preguntas la persona mayor no presentaba ninguna respuesta de manera espontánea, por lo que entonces se pasaba a ir exponiéndole las diferentes opciones de respuesta, para que ella nos dijera la que considerara oportuna. Una vez que se le presentaban todas las preguntas del cuestionario, se le daba las gracias por su colaboración.

Las respuestas dadas por cada uno de los entrevistados se escribieron de manera literal en el impreso de la entrevista correspondiente a cada uno de los participantes.

Para analizar la información recogida en las preguntas abiertas del cuestionario se ha utilizado la técnica del análisis de contenido. Con las respuestas dadas en cada una de las preguntas se han establecido diferentes categorías de respuestas.

Se trata de un estudio transversal descriptivo, donde se ha utilizado como técnica una entrevista semiestructurada de preguntas abiertas y cerradas. Cada entrevista tuvo una duración de unos 35-40 minutos aproximadamente.

Para saber si la entrevista era adecuada para nuestro estudio se hizo un juicio de expertos, mostrando dicho cuestionario a varios profesionales con experiencia en el ámbito de las personas mayores en residencias. Por otro lado, se hizo un estudio piloto de la entrevista, pasándola a un grupo de personas mayores en residencias y 
centros de día. Tras este estudio piloto se hicieron una serie de modificaciones sobre la entrevista inicial, cambiando el orden de algunas preguntas, fusionando algunas preguntas que estaban por separado en una sola, etc.

En este trabajo sólo se exponen los resultados de algunas de las cuestiones que componen la entrevista, ya que presentar todos los aspectos analizados sobrepasaría la extensión y objetivos de dicho artículo.

\section{Resultados}

\section{Actividades que se realizan en el centro/residencia:}

¿Qué actividades se llevan a cabo en el centro?

Se exponen las actividades que han señalado los participantes, con el \% de personas que las han nombrado, por lo que se considera que las que aparecen con un $\%$ más alto son las que se ofrecen en más centros/residencias, aunque estos porcentajes tendrían ciertos matices, ya que hay actividades que se ofertan y los participantes no las nombran.
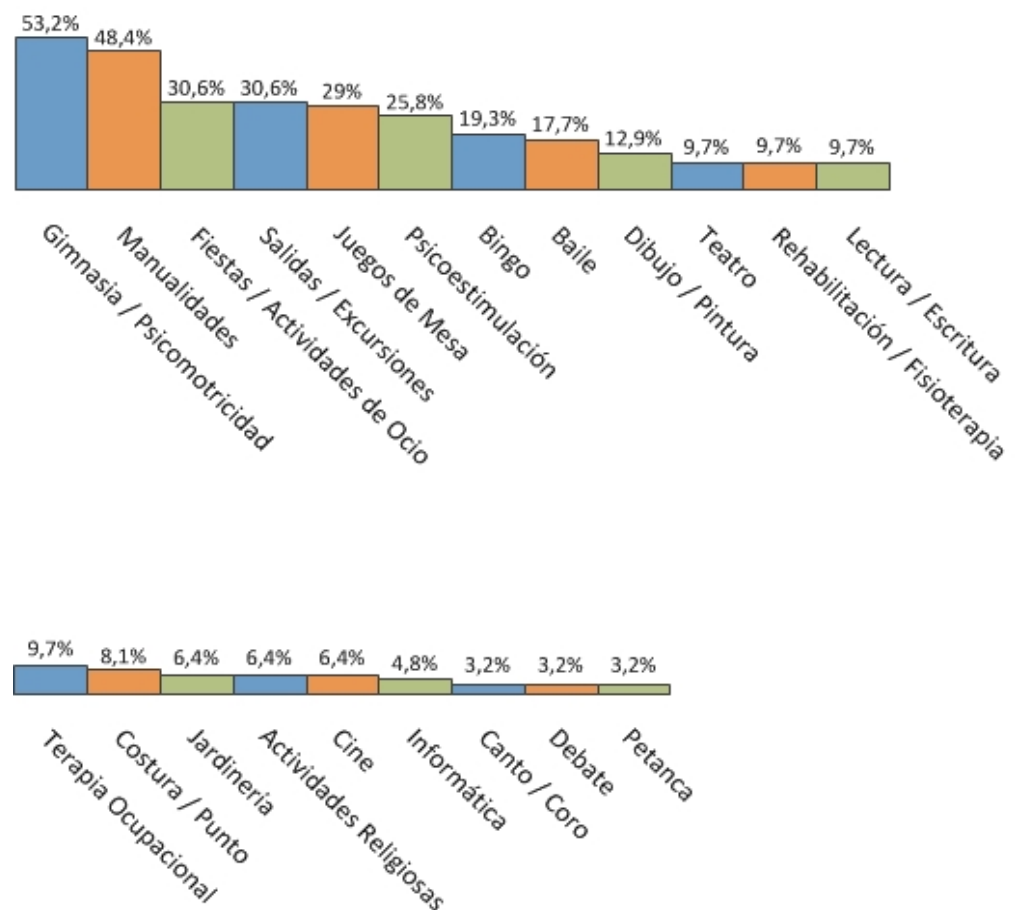

Las actividades más nombradas por los participantes son gimnasia/psicomotricidad, con un $53,2 \%$ y manualidades con un $48,4 \%$. A continuación estarían las siguientes: fiestas y actividades de ocio $(30,6 \%)$, salidas y excursiones $(30,6 \%)$, juegos de mesa (29\%), psicoestimulación $(25,8 \%)$, bingo $(19,3 \%)$, baile $(17,7 \%)$, dibujar/pintura $(12,9 \%)$

Otras actividades que mencionan los participantes que se realizan en los centros y residencias, aunque con menor frecuencia son: teatro $(9,7 \%)$, rehabilitación/fisioterapia $(9,7 \%)$, lectura, escritura y alfabetización $(9,7 \%)$, terapia ocupacional $(9,7 \%)$, costura/punto $(8,1 \%)$, huerto/jardinería $(6,4 \%)$, actividades 
religiosas $(6,4 \%)$ cine $(6,4 \%)$, informática $(4,8 \%)$, canto/coro $(3,2 \%)$, petanca $(3,2 \%$, debates/tertulias/charlas: 3,2 \%.

\section{Satisfacción con las actividades:}

¿Le agradan las actividades que se realizan? ¿Por qué?

En la pregunta acerca de si la persona estaba satisfecha con las actividades que se ofrecían en el centro un $96,8 \%$ de los participantes han respondido que sí, un 1,6\% que no y otro $1,6 \%$ que con algunas de ellas.

\section{Motivos de satisfacción:}

Algunos de los motivos por los que han expresado que estaban satisfechos han sido: "el estar ocupado, distracción, diversión, reírse, entretenimiento, ayudan a pasar el tiempo, son buenas, se trabaja la mente, hacen pensar, divertidas, me ayudan a estar mejor, estar ocupado, se aprende, estar ocupado, me gustan, sentirse útiles, las elegimos nosotros, sentirme mejor físicamente, diversión, relacionarse con compañeros, interesantes".

Una persona que responde que sí está satisfecha con las actividades ofertadas, dice que "añadiría más actividades". La persona que ha dicho que no está satisfecha, ha verbalizado: "Considero que se pierde el tiempo".

\section{Participación en las actividades:}

¿Participa en las actividades que se realizan?

Respecto a la cuestión que preguntaba a la persona sobre su participación en las actividades que se realizaban, un $61,3 \%$ ha respondido que sí, un $4,9 \%$ que no, un $14,5 \%$ que en la mayoría, un $17,7 \%$ que en algunas y un $1,6 \%$ no contestan.

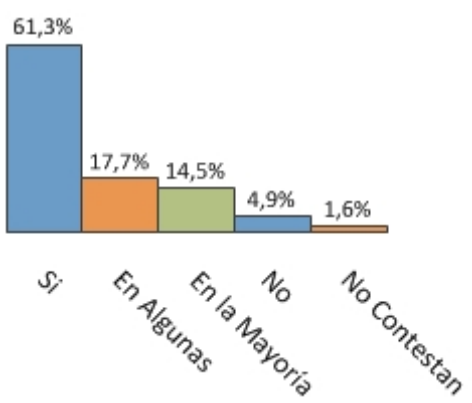

Algunos de las personas mayores especifican que no participan en todas las actividades por imposibilidad en algunos momentos (cansancio) o por dificultad física.

\section{Actividades en que participan y motivos de participación:}

¿En cuáles participa?

En la cuestión en que se preguntaba en qué actividades participaban, las respuestas dadas han sido las siguientes (por orden de mayor a menor frecuencia). 


\section{Actividades en que participan:}

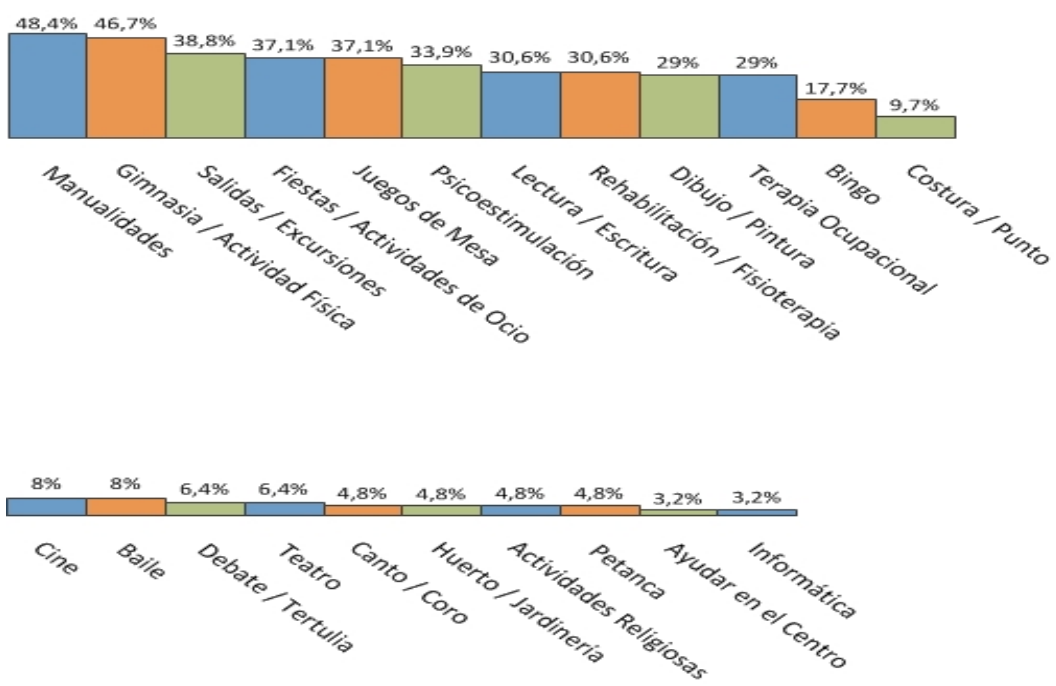

Observando los resultados vemos que las actividades de mayor participación son manualidades $(48,4 \%)$ y gimnasia $(46,7 \%)$, seguidas de salidas y excursiones $(38,8$ $\%)$, fiestas, actividades de ocio $(37,1 \%)$, juegos de mesa $(37,1)$ y psicoestimulación $(33,9 \%)$.

Tras éstas aparecen lectura y escritura $(30,6 \%)$, actividades de rehabilitación y fisioterapia (30,6 \%), dibujo y pintura (29\%), actividades de terapia ocupacional $(29 \%)$ y bingo $(17,7 \%)$.

También aparecen, aunque con una participación más baja, actividades de costura y punto, cine, baile, debates y tertulias, teatro, canto y coro, actividades de huerto y jardinería, actividades religiosas, petanca, actividades que suponen ayudar en el centro e informática.

\section{Motivos de participación:}

¿Por qué participa en estas actividades?

Respecto a los motivos de participación se han encontrado las siguientes categorías de respuesta:

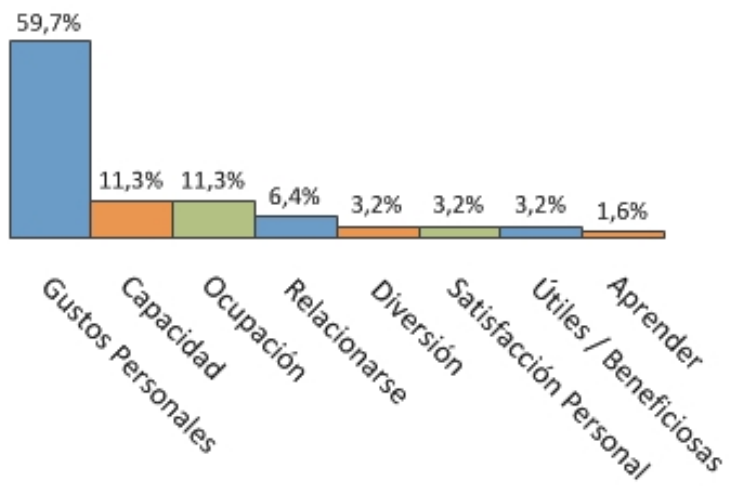


El motivo más frecuente por el que las personas mayores dicen participar en las actividades hace referencia a gustos personales y a que les resultan agradables las mismas $(59,7 \%)$. Seguido de éste motivo están los referidos a la capacidad, donde entrarían respuestas que expresan que se les da bien esa actividad, que tienen capacidad para realizarla o que la actividad les hace sentirse con capacidad $(11,3 \%)$. Con la misma frecuencia a este motivo último estaría el que expresa entretenimiento, estar ocupado o no aburrirse.

El siguiente motivo para participar en las actividades por orden de frecuencia es el que expresa estar con los demás o relacionarse $(6,4 \%)$. Otros motivos que aparecen para participar en las actividades, aunque con menos frecuencia que los anteriores son "pasarlo bien, diversión" $(3,2 \%)$, "sentirse bien con uno mismo" $(3,2 \%)$, "útiles, se obtiene algún beneficio con su realización" (3,2\%) y "aprender" (1,6\%).

\section{Actividades que le gustaría realizar:}

¿Qué actividades le gustaría realizar?

Ejercicio u otra actividad física.

Distracciones o espectáculos dentro de la Residencia (por ejemplo: actuaciones de pianistas o cantantes).

Grupos de discusión.

Grupos de orientación sobre la vida diaria.

Grupos de apoyo o autoayuda.

Cine.

Grupos de teatro, canto, rondalla u otro similares.

Conferencias o cursillos.

Bingos, juegos de cartas, otros juegos.

Fiestas.

Servicios religiosos.

Talleres de cultura general.

Trabajos manuales.

Otras: 
Actividades y $\%$ de personas mayores que las eligen:

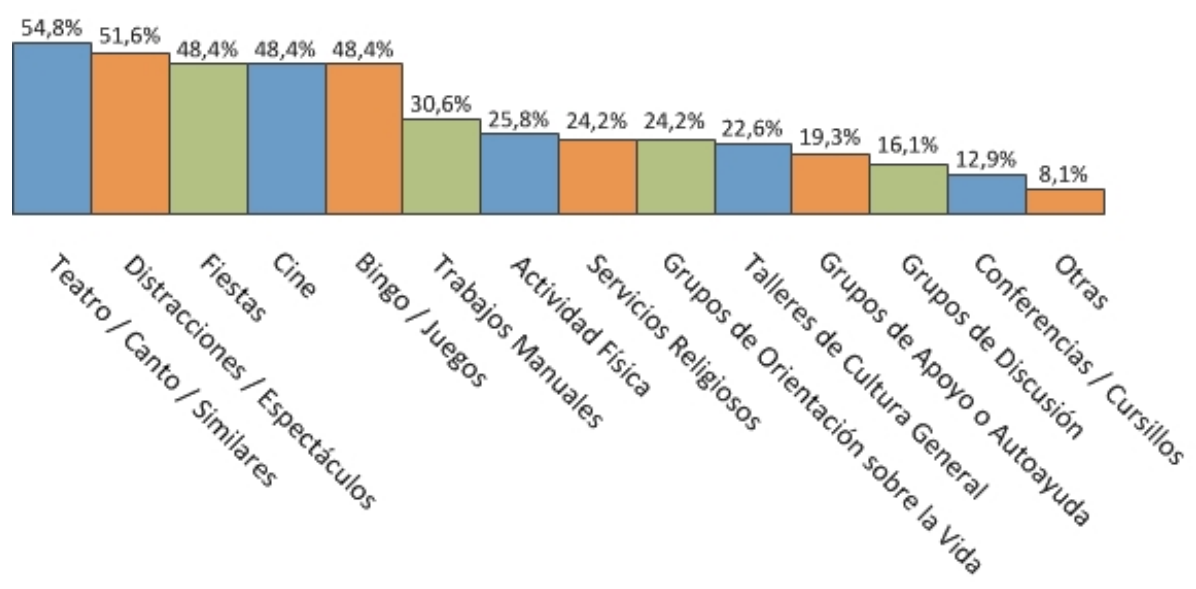

Estas actividades son las que los participantes en el estudio expresan que les gustaría realizar. La mayor parte de las personas mayores han elegidos varias actividades. Las más mencionadas son: grupos de teatro, canto, rondalla u otros equivalentes $(54,8 \%)$, distracciones o espectáculos dentro de la residencia $(51,6 \%)$, fiestas $(48,4 \%)$, cine $(48,4 \%)$, bingos, juegos de cartas y otros juegos $(48,4 \%)$.

Seguidas de éstas aparecen: trabajos manuales $(30,6 \%)$, actividad física $(25,8 \%)$, servicios religiosos $(24,2 \%)$, grupos de orientación sobre la vida $(24,2 \%)$, talleres de cultura general $(22,6 \%)$, grupos de apoyo o autoayuda (19,3\%), grupos de discusión $(16,1 \%)$, conferencias o cursillos $(12,9 \%)$ así como otras que expresan sin que aparezcan como alternativa de respuesta $(8,1 \%)$.

\section{Actividades antes de acudir al centro:}

¿Cómo ocupaba su tiempo libre antes de venir a la residencia?
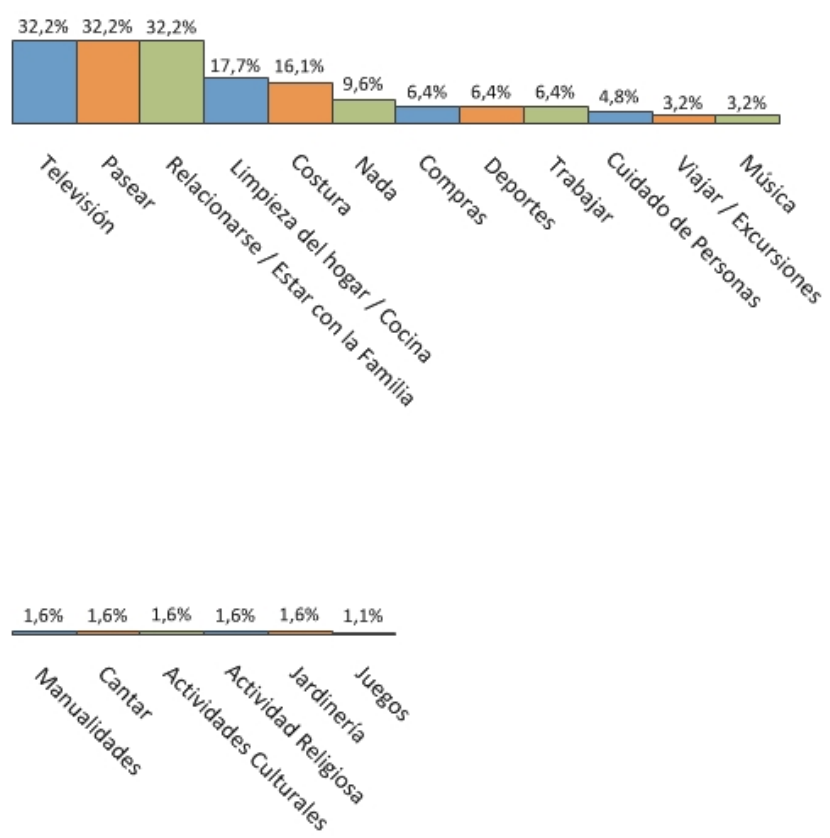
En esta cuestión la persona mayor expresaba diferentes actividades en la mayor parte de los casos. Las actividades más frecuentes en las que los mayores ocupaban el tiempo antes de acudir al centro de día o residencia son ver la televisión, pasear, relacionarse o estar con la familia o amigos/as, todas éstas en el 32,2 \% de los participantes. A continuación estarían actividades relacionadas con la limpieza y cocina $(17,7 \%)$ y con la costura $(16,1 \%)$. El $9,6 \%$ de la muestra responde que no hacía nada, con respuestas como estas: "los días eran muy largos", "deprimida, la residencia me ha dado la vida".

Actividades como comprar, hacer deporte y "trabajar" (donde en muchos casos especificaban actividades en el campo), han sido expresadas en un 6,4\% de los participantes.

Un 4,8\% expresan que parte de su tiempo lo dedican al cuidado de algún familiar, en muchos casos nietos. Otras actividades mencionadas han sido: viajar/excursiones $(3,2$ $\%)$, música $(3,2 \%)$, manualidades $(1,6 \%)$, cantar $(1,6 \%)$, actividades culturales $(1,6$ $\%)$, actividad religiosa $(1,6 \%)$, jardinería $(1,6 \%)$ y juegos $(1,1 \%)$.

\section{Estado de Ánimo más frecuente:}

¿Cómo se siente normalmente?

Alegre
Triste
Sol@
Aburrido
Tranquil@

Otra

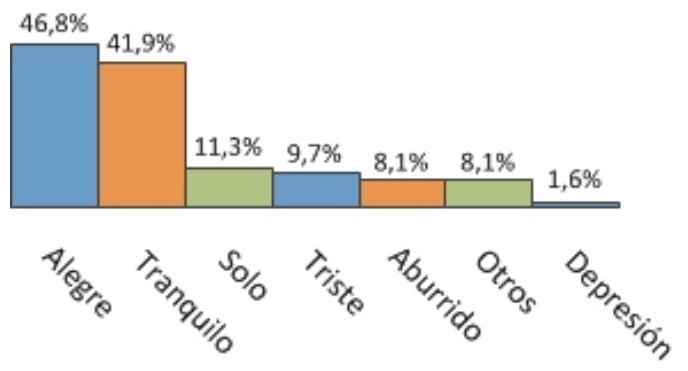

El estado de ánimo más frecuente expresado es alegre, que con un $46,8 \%$, seguido de tranquilo con un $41,9 \%$. A continuación aparecen solo $(11,3 \%)$, triste $(9,7 \%)$, otro $(8,1 \%)$, aburrido $(8,1 \%)$ y depresivo $(1,6 \%)$.

En la categoría "otro", entran estados de ánimo que pueden considerarse como más positivos ("distraída", "querida" y "ayudada") y otros menos ("dolorida"," no quiero pensar en el futuro"), pero la mayoría de las respuestas que entran en la categoría otros, son positivas. 
Hay que tener en cuenta que algunos de los participantes expresan dos estados de ánimo como los más frecuentes que suelen tener.

\section{Cosas que te podrían hacer sentir mejor:}

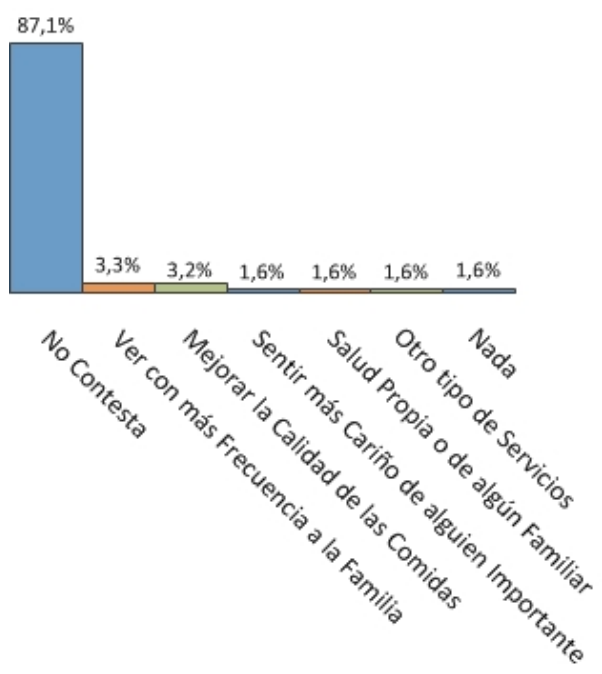

El $87,1 \%$ de la muestra no contesta a esta cuestión, un 3,3\% responde que podría hacerle sentir mejor ver con más frecuencia a su familia, el 3,2 \% mejorar la calidad de las comidas del centro, un 1,6\% la mejora de la salud propia o de algún familiar, otro $1,6 \%$ que se ofrezcan otro tipo de servicios en el centro y otro $1,6 \%$ responde explícitamente que nada.

\section{Discusión}

Nuestro interés principal era conocer si los programas de actividades que se llevan a cabo en los centros de día y residencias de mayores son los adecuados a las necesidades e intereses de las personas mayores y la forma en que se podrían mejorar estos. La mayoría de las veces los programas y actividades se realizan sin preguntar a los propios interesados y guiados por la intuición o por lo ya establecido, sin saber si se está actuando de la manera más adecuada.

Basándonos en los resultados podemos decir que en los centros y residencias se realizan diferentes tipos de actividades como gimnasia, manualidades, actividades recreativas (bingo, juegos...), lecto-escritura y talleres de cocina entre otras, pero hay actividades que se realizan en estos centros y no todos los mayores nombran.

Lo que más interesa es qué actividades tienen los mayores presentes de las que se ofertan, ya que si se hacen otras actividades y los mayores ni siquiera las nombran es que no las tienen en mente y difícilmente participarán en ellas. De ahí podemos observar la necesidad de una mayor difusión e información al usuario de estos servicios, con horarios de las diferentes actividades, recordatorios, etc.

La mayor parte de las personas mayores entrevistadas participan en algún tipo de actividad. Las actividades en que participan un mayor número de personas son la gimnasia, el bingo, las manualidades y las salidas fuera de los centros. Sin embargo, se puede observar que la participación general en las actividades es baja, ya que los $\%$ de cada actividad por separado son bajos. Por lo tanto, sería necesario tomar 
medidas para aumentar la participación en las actividades que se ofertan, aumentar la motivación de los mayores y ofrecer actividades más variadas.

Las actividades de mayor participación implican creatividad, movimiento, contacto con el exterior, salir del centro, trabajar en grupo, relacionarse y estimulación de funciones cognitivas y físicas, por lo que se deberían de ofrecer más actividades al aire libre, que sean grupales, participativas, que supongan movilización, estimulación, contacto, etc.

Habría que analizar con más detenimiento si son las de mayor participación porque son las que se ofrecen en un mayor número de centros o porque son las que los mayores más demandan. No todas las actividades que los mayores mencionan se ofrecen en todos los centros.

Hay que tener en cuenta que las personas con deterioro cognitivo importante no han sido entrevistadas. La mayoría de estas personas participan en un número muy limitado de actividades o en casi ninguna, dependiendo del grado del deterioro. De todos modos, no se debe de olvidar el ofrecer actividades alternativas acordes a sus necesidades y adaptadas a sus déficits.

Considerando las actividades socioculturales o educativas exclusivamente y dejando las rehabilitadoras o sanitarias a un lado, vemos que los mayores participan en actividades como manualidades, gimnasia, salidas y excursiones, fiestas y actividades de ocio, juegos de mesa, psicoestimulación, lectura y escritura y bingo entre otras. Como vemos la mayoría se realizan con otros compañeros, lo que supone compartir con los demás, relacionarse, sentirse parte de un grupo, etc.

Respecto a la satisfacción de los mayores con estas actividades se puede decir que son de la satisfacción de la mayoría de las personas mayores entrevistadas, aunque sería necesario introducir algunos cambios, como adaptarlas a la capacidad de la persona mayor y ampliar la variedad de actividades para que puedan ser del interés de todos, además de que se realicen con mayor frecuencia actividades y principalmente las más demandadas por los mayores.

El motivo más expresado por las personas mayores para participar en las actividades ha sido "me gusta", haciendo referencia a gustos personales. Otras razones también expresadas han sido: sentirse bien, compartir con los demás, sentirse con capacidad para llevar a cabo la actividad, entretenimiento, relacionarse con los demás, diversión, sentirse bien con uno mismo, considerarlas útiles, obtener algún beneficio y aprender.

Tras las respuestas dadas por las personas mayores en cuanto a los motivos de la participación en las actividades, se pueden desprender los siguientes beneficios que encuentran en las actividades en que participan: mejorar el estado de ánimo, mejorar la autoestima (sentirse capaz de realizarlas, etc.), relacionarse con los demás, mantenerse activo, mantener las capacidades físicas e intelectuales. Conociendo los beneficios que les aportan las actividades en que participan, los programas de actividades deberían de ir en la línea de aportar estos beneficios, lo que aumentaría la motivación de las personas mayores para participar en los mismos.

Al preguntar a los mayores sobre qué actividades nuevas les gustaría realizar, han sido diversas las que han mencionado. Entendemos que serían actividades que se podrían ofrecer desde estos recursos. Las de mayor preferencia han sido: actividades relacionadas con el teatro y la música, espectáculos, fiestas, cine, bingo, trabajos 
manuales, actividad física, servicios religiosos, grupos de orientación sobre la vida, actividades culturales, grupos de apoyo o autoayuda y grupos de discusión entre otras.

Algunas de estas actividades se ofrecen en algunos de los centros y han sido mencionadas por algunos de los participantes al preguntar por las actividades que se llevan a cabo en el centro o actividades en que participan (fiestas, cine, bingos, trabajos manuales, actividad física y servicios religiosos), mientras que otras de las que les gustaría realizar, no se ofertan en la mayoría de los centros y residencias. Podemos considerar que si estas actividades se ofrecieran en estos servicios, serían actividades de alta participación, ya que irían en consonancia con las preferencias de las personas mayores.

Parece que en general la actividad de los mayores antes de acudir a estos servicios era menor o similar. Recordemos que un $9,6 \%$ de los participantes han respondido que antes de acudir al centro o residencia no hacían nada. Por otro lado, la mayor parte ocupaba su tiempo viendo la televisión, paseando o relacionándose con amigos o familiares. Por tanto, se debe de tener presente que en un alto porcentaje de los participantes, el acudir al centro de día o residencia ha supuesto un incremento de su actividad. Sin embargo echan de menos algunas cosas de su vida antes de acudir a la residencia como compartir más tiempo con sus familiares, vecinos y amigos o estar en su casa en el caso de las personas que están en residencia. De ahí la importancia de que además de poder hacer nuevas actividades que les resulten positivas, puedan seguir manteniendo actividades que realizaban antes, así como mantener el mayor contacto posible con sus familiares y amigos.

Aproximadamente la mitad de los participantes dicen encontrarse con un buen estado de ánimo. Entre los estado menos positivos están además de la tristeza, la soledad, de ahí la necesidad de mejorar el sentimiento de formar parte de un grupo, de aumentar los contactos sociales, familiares, etc.

Consideramos muy importante favorecer la mayor continuidad entre la vida que la persona mayor llevaba antes de acudir al centro o residencia, especialmente en este último recurso, donde hay mayor riesgo de institucionalización y de pérdidas a diferentes niveles. El centro de día tiene la ventaja de favorecer esta continuidad, ya que el usuario pasa parte del día en su domicilio.

Para la persona mayor se ha podido ver que es muy importante sentirse con capacidad, apoyo familiar y social, por lo que se podrían aumentar las actividades en que participaran familiares, así como que les hagan sentirse con capacidades y con un sentimiento de estar haciendo algo útil.

Hay que destacar la poca esperanza respecto al futuro que se ha podido apreciar por las pocas respuestas dadas en el ítem acerca de qué podría hacerte sentir mejor. Se observa con ello que hay pocos objetivos vitales de mejora o poca esperanza de cambio. Sería necesario fomentar actividades dirigidas a mejorar este aspecto.

Por otro lado, sería necesario facilitar la corrección de los déficits sensoriales que presente la persona o adaptar y modificar los materiales para que estos no sean un obstáculo para la realización de la tarea.

Una posibilidad que se podría considerar sería el realizar varias actividades al mismo tiempo, con pequeños grupos y una pequeña supervisión de algunas de las 
actividades por un profesional, para que mientras unos mayores participan en algo otros puedan estar con otra actividad más cercana a sus gustos y así poder hacer con más frecuencia actividades que los mayores demandan más.

Consideramos que sería necesario la realización al inicio del ingreso de una entrevista con el usuario, para conocer sus preferencias y gustos personales y actividades que realizaba antes de acudir al centro para favorecer la mayor continuidad posible.

\section{Referencias}

Bazo, MT. La sociedad anciana. Madrid: Siglo XXI, 1992.

Bazo, MT. Aportaciones de las personas mayores a la sociedad: Análisis sociológico. Revista Española de Investigaciones Sociológicas, 73, 209-222. Centro de Investigaciones Sociológicas: Madrid, 2006.

Bedmar, M. y Montero, I. (2009). Recreando la Educación en Personas Mayores: aportes desde la Pedagogía Social. Granada: Grupo Editorial Universitario.

Fernández-Ballesteros, R. (Dir.) (1996): Sistema de Evaluación de Residencias de Ancianos (SERA).Madrid: INSERSO.

Fernández-Ballesteros, R.; Zamarrón, M.D. y Maciá, A. (1996): Calidad de vida en la vejez en distintos contextos. Madrid: INSERSO.

Fernández Ballesteros R. Mitos y realidades sobre la vejez y la salud. Fundación Caja Madrid: Barcelona, 1992.

Fernández Ballesteros R Gerontología social. Pirámide, Colección Psicología: Madrid, 2000.

Kaufmann, A.E. y otros. "Residencias: lo público y lo privado". REIS N73. CIS, 1996.

Rodríguez, P. "La Residencia. Concepto, destinatarios y objetivos generales", en Pilar Rodríguez Rodríguez, Residencias para personas mayores, Editorial Médica Panamericana, Madrid. 1999. 